\title{
A Facile Ionic Liquid Promoted Synthesis, Cholinesterase Inhibitory Activity and Molecular Modeling Study of Novel Highly Functionalized Spiropyrrolidines
}

\section{Abdulrahman I. Almansour ${ }^{1}$, Raju Suresh Kumar ${ }^{1, *}$, Natarajan Arumugam ${ }^{1}$, Alireza Basiri ${ }^{2}$, Yalda Kia $^{3}$, Mohamed Ashraf Ali ${ }^{4}$, Mehvish Farooq ${ }^{5}$ and Vikneswaran Murugaiyah ${ }^{2}$}

1 Department of Chemistry, College of Science, King Saud University, P.O. Box 2455, Riyadh 11451, Saudi Arabia; E-Mails: almansor@ksu.edu.sa (A.I.A.); aruorgchem@gmail.com (N.A.)

2 School of Pharmaceutical Sciences, Universiti Sains Malaysia, Minden 11800, Malaysia; E-Mails: diba115920@gmail.com (A.B.); vicky@usm.my (V.M.)

3 School of Chemical Sciences, Universiti Sains Malaysia, Minden 11800, Malaysia; E-Mail:kia.yalda@yahoo.com

4 Pharmacogenetic and Novel Therapeutic Institute for Research in Molecular Medicine, Universiti Sains Malaysia, Minden 11800, Malaysia; E-Mail: asraf80med@yahoo.com

5 Institute of Pharmaceutical Science, King's College London, London SE1 9NH, UK; E-Mail: Mehvish.farooq@kcl.ac.uk

* Author to whom correspondence should be addressed; E-Mail: sraju@ksu.edu.sa or drrajusureshkumar@gmail.com; Tel.: +966-4675907; Fax: +966-4675992.

Academic Editor: Jean Jacques Vanden Eynde

Received: 17 November 2014 / Accepted: 23 January 2015 / Published: 29 January 2015

Abstract: A series of novel dimethoxyindanone embedded spiropyrrolidines were synthesized in ionic liquid, [bmim] $\mathrm{Br}$ and were evaluated for their inhibitory activities towards cholinesterases. Among the spiropyrrolidines, compound $\mathbf{4 f}$ exhibited the most potent activity with an $\mathrm{IC}_{50}$ value of $1.57 \mu \mathrm{M}$ against acethylcholinesterase (AChE). Molecular docking simulation for the most active compound was employed with the aim of disclosing its binding mechanism to the active site of AChE receptor.

Keywords: acetylcholinesterase (AChE), butyrylcholinesterase (BChE), Alzheimer's disease (AD), spiropyrrolidines, ionic liquid and molecular docking study 


\section{Introduction}

Alzheimer's disease (AD), the most common cause of dementia, is a neurodegenerative disorder characterized by progressive decline of memory and cognition [1]. One of the major therapeutic strategies adopted for primarily symptomatic AD is based on the cholinergic hypothesis targeting cholinesterase enzymes [2]. Inhibition of the hydrolysis of acetylcholine (ACh) by blocking its metabolic enzyme acetylcholinesterase (AChE) increases the ACh concentration and provides a possible symptomatic treatment option for $\mathrm{AD}$. On the other hand, butyrylcholinesterase (BChE) has recently been considered as a potential target because it also plays an important role in regulating ACh level [3]. Although there are many ongoing research activities in the search of drugs for treating $\mathrm{AD}$, only four drugs are currently approved for treatment and these drugs do not show potential cure rates. However, from academic and practical standpoints, it is still desirable to develop more effective agents for the treatment of $\mathrm{AD}$. The increasing mortality rate and the reduced therapeutic potential of the currently available options have led us to focus our research activities on the development of potential cholinesterase inhibitors for the treatment of AD.

Among the five-membered heterocyclic systems, spiropyrrolidines occupy an important place as this structural motif appears as an integral part of many natural products besides exhibiting anticonvulsant [4-6], potential antileukemic [7], local anaesthetic [8] and antiviral activities [9]. Ionic liquids are widely recognized as "green" solvents in organic synthesis because of their unique properties, such as low vapor pressure, high chemical and thermal stability, solvating ability, non-flammability, behavior as acidic or basic catalysts and recyclability [10-14]. In this context, ionic liquids have emerged as new green solvents to replace the volatile organic compounds and they are found suitable for executing many diverse organic reactions [15-17]. The development of multi-component reactions in ionic liquids, although relatively unexplored [18-22] is of great interest and to the best of our knowledge, this is the first report on 1,3-dipolar cycloaddition reaction of 2-arylmethylidene-5,6-dimethoxy-2,3-dihydro- $1 H$-inden-1-one with an unexplored and new class of azomethine ylide generated in situ from $L$-phenylalanine and isatin in ionic liquid medium, namely 1-butyl-3-methylimidazolium bromide ([bmim]Br).

As a part of our ongoing research project aimed at identifying potential cholinesterase inhibitors for AD therapy [23-33], we report here our efforts in search of novel potent cholinesterase inhibitors using dipolar cycloaddition strategy. In addition, molecular modeling studies were also performed to disclose the binding modes of the most active inhibitors to the amino acid residues that compose the active site of the AChE enzyme.

\section{Results and Discussion}

\subsection{Chemistry}

The target spiropyrrolidines $\mathbf{4 a}-\mathbf{j}$ were synthesized as outlined in Scheme 1. The required starting precursors 2-arylmethylidene-5,6-dimethoxy-2,3-dihydro-1H-inden-1-ones $\mathbf{1 a}-\mathbf{j}$ were synthesized following the synthetic protocol reported earlier by us [34] by the reaction of 5,6-dimethoxy-1indanone with appropriate aromatic aldehydes in dilute methanolic sodium hydroxide. The three component 1,3-dipolar cycloaddition reaction of a series of $\mathbf{1 a}-\mathbf{j}$ with a new class of relatively 
unexplored azomethine ylide generated in situ from isatin (2) and phenylalanine (3) in [bmim]Br afforded the functionalized spiropyrrolidines $\mathbf{4 a - j}$ in good yields.

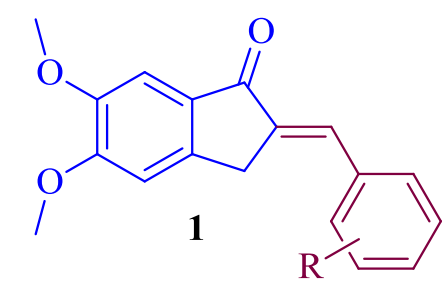

MeOH<smiles>COc1cc2c(cc1OC)C(=O)CC2</smiles><smiles>O=C1Nc2ccccc2C1=O</smiles><smiles>NC(Cc1ccccc1)C(=O)O</smiles>

3

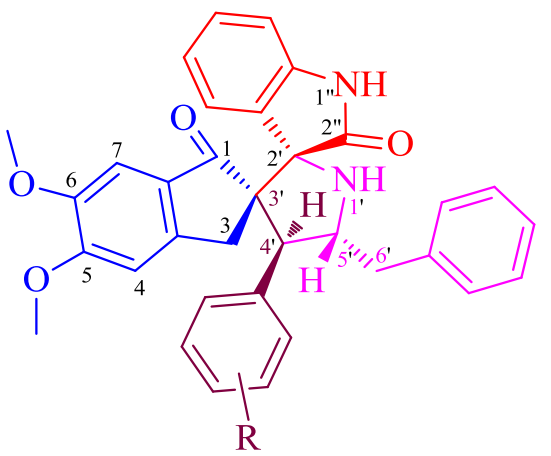

4 (10 examples)

1a and $4 \mathrm{a}, \mathrm{R}=\mathrm{H} ; \quad \mathbf{1 b}$ and $4 \mathbf{b}, \mathrm{R}=2-\mathrm{CH}_{3}$;

$1 \mathbf{c}$ and $\mathbf{4 c}, \mathrm{R}=2-\mathrm{OCH}_{3} ; \mathbf{1 d}$ and $\mathbf{4 d}, \mathrm{R}=2-\mathrm{Cl}$;

1e and $4 \mathbf{e}, \mathrm{R}=3-\mathrm{OCH}_{3} ; \mathbf{1 f}$ and $4 \mathrm{f}, \mathrm{R}=3-\mathrm{O}_{2} \mathrm{~N}$;

$1 \mathrm{~g}$ and $4 \mathrm{~g}, \mathrm{R}=4-\mathrm{CH}_{3} ; \quad \mathbf{h}$ and $4 \mathrm{~h}, \mathrm{R}=4-\mathrm{OCH}_{3}$;

$\mathbf{1 i}$ and $4 \mathbf{i}, \mathrm{R}=4-\mathrm{Cl} ; \quad \mathbf{1 j}$ and $\mathbf{4 j}, \mathrm{R}=2,4-\mathrm{Cl}_{2}$

Scheme 1. Synthesis of spiropyrrolidines $\mathbf{4 a}-\mathbf{j}$.

Solvent optimization for this cycloaddition reaction was investigated by choosing the model reaction between azomethine ylide and 2-(2-methylphenyl)methylidene-5,6-dimethoxy-2,3-dihydro$1 H$-inden-1-one (1) $)$. The reaction of an equimolar mixture of $\mathbf{1 b}$, isatin and phenylalanine in refluxing methanol, methanol:dioxane and dioxane for $5 \mathrm{~h}$ afforded the spiropyrrolidine (4b) in $44 \%$, $52 \%$ and $58 \%$ yield whilst the reaction in [bmim] $\mathrm{Br}$ furnished $\mathbf{4 b}$ in an excellent yield of $87 \%$ in a short reaction time of $1 \mathrm{~h}$, suggesting [bmim] $\mathrm{Br}$ as the appropriate solvent in terms of enhanced yield and reaction time for the cycloaddition reactions of this kind. Having determined the optimal conditions, all the subsequent reactions were effected by heating an equimolar mixture of the reactants in [bmim] $\mathrm{Br}(200 \mathrm{mg})$ in an oil-bath at $100{ }^{\circ} \mathrm{C}$ for $1 \mathrm{~h}$. After completion of the reaction as evidenced by TLC, the product was isolated and purified through column chromatography, whilst the ionic liquid is recovered and reused, its efficacy being significantly undiminished in subsequent runs. As shown in Table 1, the cycloaddition reaction works well regardless of the position and electronic or steric properties of the substituents at the aromatic ring of compound $\mathbf{1}$ affording the cycloadduct $\mathbf{4}$ in good yields.

The structure of spiropyrrolidines $\mathbf{4}$ was elucidated unambiguously with the help of one and two-dimensional NMR spectroscopic studies. As a representative case the chemical shift assignments of $\mathbf{4 b}$ are discussed. In the ${ }^{1} \mathrm{H}-\mathrm{NMR}$ spectra of $\mathbf{4 b}$, the doublet at $4.10 \mathrm{ppm}(\mathrm{J}=8.8 \mathrm{~Hz}) \mathrm{can}$ be assigned to H-4'. H,H-COSY correlation of H-4' assigns the multiplet at 4.95-5.01 ppm to H-5'. The C,H-COSY correlation of H-4' and H-5' assigns the carbon signals at 55.44 and 64.85 ppm to C-4' and C-5', respectively. Further, the H,H-COSY correlation of H-5' assigns the doublet of doublets at 2.93 $(J=14.0,8.8 \mathrm{~Hz})$ and $3.02(J=14.0,4.4 \mathrm{~Hz})$ to the benzylic protons $\left(6 '-\mathrm{CH}_{2}\right)$. The C,H-COSY correlation of $6^{\prime}-\mathrm{CH}_{2}$ assigns the carbon signal at $40.45 \mathrm{ppm}$ to $\mathrm{C}-6$ '. The remaining doublets at 2.62 
and $2.73 \mathrm{ppm}(J=18.3 \mathrm{~Hz})$ are due to $3-\mathrm{CH}_{2}$. Further, $\mathrm{H}-4$ ' shows $\mathrm{HMBC}$ with the $\mathrm{C}=\mathrm{O}$ of indanone ring, C-5', C-6', C-3 besides showing correlation with the aromatic ring carbons. The carbon signals at 66.69 and $75.08 \mathrm{ppm}$ are due to the spiro carbons C-3' and C-2', respectively. The carbon chemical shifts of C-3, C-4', C-5' and C-6' were also confirmed from DEPT 135 NMR spectrum (vide supplementary Materials).The singlets at 1.92, 3.70, 3.80 and $8.01 \mathrm{ppm}$ can be attributed to the methyl, two methoxy of aromatic rings and the $\mathrm{NH}$ group of oxindole ring, respectively. The aromatic protons appear as singlets, doublets and multiplets around 6.19-8.05 ppm. The signals at 179.63 and $207.10 \mathrm{ppm}$ are due to the carbonyl groups of oxindole and indanone ring, respectively. The structure of other spiropyrrolidines was also assigned by similar straightforward considerations. The stereochemistry of compound $\mathbf{4 b}$ has been arrived from its NOESY spectra.

Table 1. Physical data, AChE and BChE inhibitory activities of $\mathbf{4 a}-\mathbf{j}$.

\begin{tabular}{|c|c|c|c|c|c|c|}
\hline Entry & Comp 4 & $\mathbf{R}$ & Yield $(\%)^{a}$ & $\mathbf{m p}{ }^{\circ} \mathrm{C}$ & $\begin{array}{c}\text { AChE Inhibition } \\
\text { IC }_{50} \mu \mathrm{M}( \pm \mathrm{SD})\end{array}$ & $\begin{array}{c}\text { BChE Inhibition } \\
\text { IC }_{50} \mu \mathrm{M}( \pm \mathrm{SD}) \\
\end{array}$ \\
\hline 1 & $\mathbf{a}$ & $\mathrm{H}$ & 85 & $134-135$ & $11.29 \pm 0.21$ & $15.73 \pm 0.22$ \\
\hline 2 & $\mathbf{b}$ & $2-\mathrm{CH}_{3}$ & 87 & $141-142$ & $10.15 \pm 0.17$ & $21.32 \pm 0.19$ \\
\hline 3 & c & $2-\mathrm{OCH}_{3}$ & 82 & $126-127$ & $12.23 \pm 0.19$ & $11.39 \pm 0.12$ \\
\hline 4 & d & $2-\mathrm{Cl}$ & 85 & $136-137$ & $15.17 \pm 0.22$ & $9.63 \pm 0.15$ \\
\hline 5 & $\mathbf{e}$ & $3-\mathrm{OCH}_{3}$ & 84 & $127-128$ & $2.13 \pm 0.11$ & $17.44 \pm 0.23$ \\
\hline 6 & f & $3-\mathrm{O}_{2} \mathrm{~N}$ & 89 & $133-134$ & $1.57 \pm 0.09$ & $15.32 \pm 0.18$ \\
\hline 7 & g & $4-\mathrm{CH}_{3}$ & 92 & $138-139$ & $7.11 \pm 0.13$ & $10.46 \pm 0.24$ \\
\hline 8 & h & $4-\mathrm{OCH}_{3}$ & 80 & $129-130$ & $3.67 \pm 0.15$ & $14.69 \pm 0.17$ \\
\hline 9 & $\mathbf{i}$ & 4-Cl & 88 & $132-133$ & $5.83 \pm 0.20$ & $16.11 \pm 0.15$ \\
\hline 10 & $\mathbf{j}$ & $2,4-\mathrm{Cl}_{2}$ & 90 & $135-136$ & $6.72 \pm 0.18$ & $12.79 \pm 0.25$ \\
\hline 11 & Galantamine & - & - & - & $2.09 \pm 0.07$ & $19.21 \pm 0.12$ \\
\hline 12 & Donepezil & - & - & - & $0.21 \pm 0.02$ & $3.6 \pm 0.11$ \\
\hline
\end{tabular}

a Isolated yield.

A mechanism proposed to rationalize the formation of $\mathbf{4}$ is summarized in Scheme 2. Hydrogen atom of $[\mathrm{bmim}]^{+}$being electron-deficient could form hydrogen bond between the imidazole ring hydrogen atom of [bmim $]^{+}$and the carbonyl group of isatin facilitating the attack of lone pair of phenylalanine on isatin and subsequent dehydration to furnish azomethine ylide 5. In the same way, hydrogen atom of $[\mathrm{bmim}]^{+}$forms hydrogen bond with the carbonyl group of 2-arylmethylidene-5,6dimethoxy-2,3-dihydro- $1 H$-inden-1-one providing the easy addition of azomethine ylide to the more electron deficient $\beta$-carbon to afford spiropyrrolidine 4 , regio- and stereoselectively.

The presence of $\mathrm{NH}$ and benzyl groups in adjacent positions of the pyrrolidine ring of $\mathbf{4}$ prompted us to explore their further reaction with paraformaldehyde (Pictet-Spengler cyclisation) in the presence of trifluoroacetic acid to synthesize novel isoquinoline heterocyclic hybrids. Thus, the spiropyrrolidine (4b) $(1 \mathrm{mmol})$ was treated overnight with paraformaldehyde $(1 \mathrm{mmol})$ in the presence of a catalytic amount of trifluoroacetic acid (10 mol \%) in $\mathrm{CH}_{2} \mathrm{Cl}_{2}$ at room temperature (Scheme 3). This reaction afforded the mixture of products with same $\mathrm{R}_{\mathrm{f}}$ value, which could not be separated through column chromatography. 


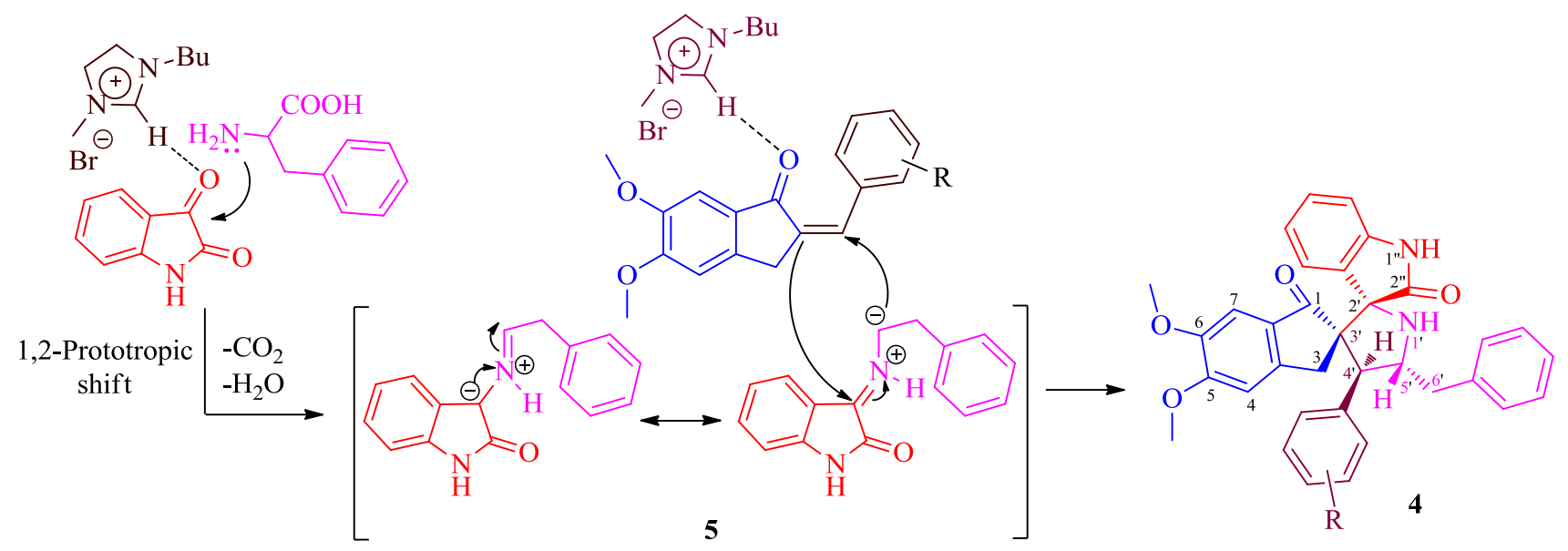

Scheme 2. Plausible mechanism for the formation of spiropyrrolidine 4.
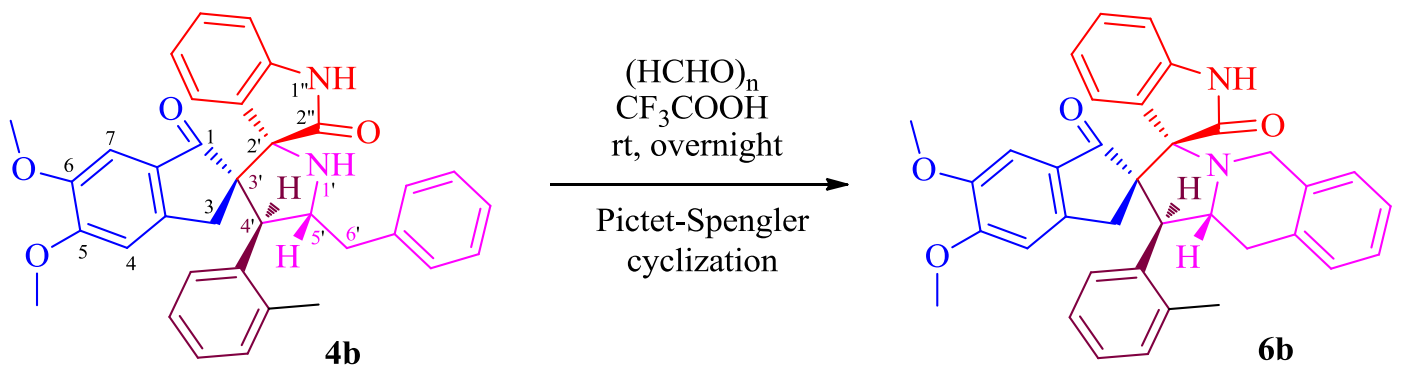

Scheme 3. Pictet-Spengler cyclisation of spiropyrrolidine $\mathbf{4 b .}$

\subsection{Cholinesterase Inhibitory Activity Study}

All newly synthesized compounds were evaluated in vitro for their cholinesterase inhibitory activity against AChE enzyme from electric eel and BChE enzyme from equine serum. As summarized in Table 1, compound $\mathbf{4 f}$, bearing nitro moiety at meta position of aromatic ring displayed highest AChE inhibitory activity with calculated $\mathrm{IC}_{50}$ value of $1.57 \mu \mathrm{M}$, followed by $4 \mathrm{e}\left(\mathrm{Ar}=m-\mathrm{OCH}_{3}\right)$, 4h $\left(\mathrm{Ar}=p-\mathrm{OCH}_{3}\right), \mathbf{4 i}(\mathrm{Ar}=p-\mathrm{Cl}), \mathbf{4 j}\left(\mathrm{Ar}=o, p-\mathrm{Cl}_{2}\right)$ and $\mathbf{4 g}\left(\mathrm{Ar}=p-\mathrm{CH}_{3}\right)$ all displaying remarkable $\mathrm{IC}_{50}$ values of lower than $10 \mu \mathrm{M}$. The rest of the compounds $4 \mathbf{a}-\mathbf{d}$ displayed moderate AChE inhibitory activities ranging from 10.15-15.17 $\mu \mathrm{M}$. From Table 1, it is clear that the presence of electron withdrawing nitro moiety, had greater impact on the AChE inhibitory potencies compared to electron donating moieties such as methoxy (strong electron donor), methyl and chloro (mild electron donor).

The active site of AChE enzyme is located at bottom of a narrow channel composed of amino acid residues with aromatic side chains such as tryptophan and tyrosine. The potency of inhibitors is closely related to the ease of insertion into this channel and their ability to interact with its composing residues. In fact, inhibitors having more facilitated insertion and better interactions with this gorge should possess higher enzyme inhibitory potentials. Thus, the inhibitor $4 \mathrm{f}$, owing to the unique electronic withdrawing nature of its nitro group, strongly binds to the AChE active site and effectively inhibited choline substrate hydrolysis. Interestingly, compounds bearing a strong electron donor methoxy moiety, also showed good inhibitory activities but at much lower degree when compared to those containing a nitro group. 
It is also worth to mention that these newly compounds are benefited from their specific chemical structure of including three aromatic cores, by showing moderate to weak BChE inhibitory potencies with calculated $\mathrm{IC}_{50}$ values ranging from $9.63-21.32 \mu \mathrm{M}$ and good selectivity toward AChE enzyme. The aromatic core multiplicity effect explains how compounds bearing three aromatic cores have higher AChE inhibitory potencies than their analogs with only two aromatic cores and introduces the aromatic content of inhibitor as an important factor to design potent AChE inhibitors [28].

Compared to standard drugs, galantamine and donepezil, compound $\mathbf{4 f}$ displayed significantly higher and compound $4 \mathbf{e}$ showed comparable AChE inhibitory activity to galantamine. None of the inhibitors had comparable activity to donepezil, due to its different planar chemical structure. For $\mathrm{BChE}$, except for compound $\mathbf{4 b}$, all the inhibitors showed higher activities than galantamine. As expected, none of the compounds showed comparable BChE activity to donepezil.

\subsection{Molecular Docking Studies}

The most active AChE inhibitor, compound $\mathbf{4 f}$ was docked into the active site of AChE enzyme derived from the crystal structure of Torpedo californica in complex with donepezil (Figure 1). Molecular docking analysis clearly demonstrated the influence of aromatic core multiplicity in the remarkable activity observed for this compound. Compound $4 \mathbf{f}$ strongly bounded to the residues having aromatic side chains such as Trp279, Tyr70, Tyr334 and Phe331, which compose peripheral anionic site (PAS) of the AChE enzyme through hydrophobic interactions took place dominantly between their aromatic entities. It was proposed that deposition of neurotoxin amyloid plaque in $\mathrm{AD}$ could be accelerated or even triggered by interaction of $\beta$-amyloids with the peripheral anionic site of the enzyme. Therefore, PAS inhibitors not only symptomatically improve the AD by blocking active site gorge entrance and prevention of acetylcholine hydrolysis, but also can have effective disease modifying properties [35].
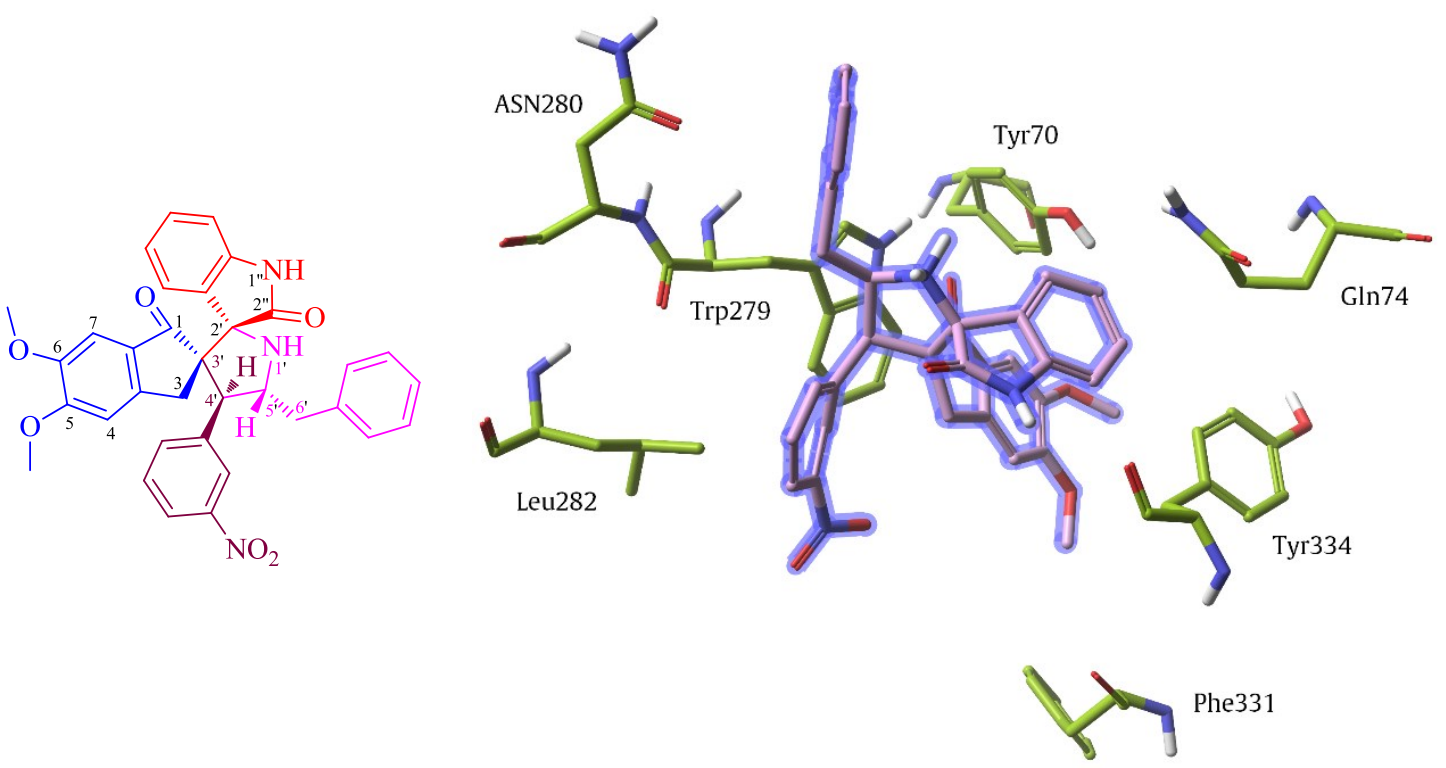

Figure 1. Schematic representation of compound $\mathbf{4} \mathbf{f}$ bounded to active site of AChE enzyme. 


\section{Experimental Section}

\subsection{General Methods}

Melting points were measured using open capillary tubes and are uncorrected. ${ }^{1} \mathrm{H}$ - and ${ }^{13} \mathrm{C}-\mathrm{NMR}$ spectra were recorded on Jeol 500 and $400 \mathrm{MHz}$ instruments (Tokyo, Japan) in $\mathrm{CDCl}_{3}$ using TMS as internal standard. Chemical shifts are given in parts per million ( $\delta$ scale) and coupling constants are given in Hertz. IR spectra were recorded on a PerkinElmer spectrum 100 FT-IR instrument $(\mathrm{KBr}$ pellet, Shelton, AL, USA). Elemental analyses were performed 10 on a PerkinElmer 2400 Series II Elemental CHNS analyzer (Waltham, MA, USA). Column chromatography was performed on silica gel (230-400 mesh) using petroleum spirit/ethyl acetate as eluent.

\subsection{General Procedure for the Synthesis of $\mathbf{4}$}

An equimolar mixture of 2-arylmethylidene-5,6-dimethoxy-2,3-dihydro-1 $H$-inden-1-one (1), isatin (2) and phenylalanine (3) was heated with stirring at $100{ }^{\circ} \mathrm{C}$ in $200 \mathrm{mg}$ of [bmim]Br for $1 \mathrm{~h}$. After completion of the reaction (TLC), ethyl acetate $(10 \mathrm{~mL})$ was added and the reaction mixture stirred for $15 \mathrm{~min}$. The ethyl acetate layer was separated, washed with water and dried. The product obtained in good yield was purified through column chromatography. [bmim]Br after extraction of the product was completely dried under vacuum and reused for subsequent reactions.

\subsection{Characterization Data for Compounds $\mathbf{4 a - j}$}

5'-Benzyl-5, 6-dimethoxy-2,3-dihydro-4'-phenyldispiro[1H-indene-2,3'-pyrrolidine-2',3"-indoline]-

1,2"-dione (4a). IR (KBr) $v_{\max } 3405,1721,1685,1620,1594 \mathrm{~cm}^{-1} ;{ }^{1} \mathrm{H}-\mathrm{NMR}\left(400 \mathrm{MHz}, \mathrm{CDCl}_{3}\right): \delta_{\mathrm{H}}$ $2.65\left(\mathrm{~d}, J=17.6 \mathrm{~Hz}, 1 \mathrm{H}, 3-\mathrm{CH}_{2}\right), 2.86-2.97\left(\mathrm{~m}, 2 \mathrm{H}, 3-\mathrm{CH}_{2}\right.$ and $\left.6^{\prime}-\mathrm{CH}_{2}\right), 3.04-3.08\left(\mathrm{~m}, 1 \mathrm{H}, 6{ }^{\prime}-\mathrm{CH}_{2}\right)$, $3.65\left(\mathrm{~s}, 3 \mathrm{H}, \mathrm{OCH}_{3}\right), 3.78\left(\mathrm{~s}, 3 \mathrm{H}, \mathrm{OCH}_{3}\right), 3.94\left(\mathrm{~d}, J=8.8 \mathrm{~Hz}, 1 \mathrm{H}, \mathrm{H}-4{ }^{\prime}\right), 4.70-4.81$ (m, 1H, H-5'), 6.19 (s, 1H, Ar-H), 6.58 (d, J=7.2 Hz, 1H, Ar-H), 6.86-7.40 (m, 14H, Ar-H), 8.37 (s, 1H, NH). ${ }^{13} \mathrm{C}-\mathrm{NMR}$ $\left(100 \mathrm{MHz}, \mathrm{CDCl}_{3}\right)$ : $\delta_{\mathrm{C}} 34.27(\mathrm{C}-3), 40.10\left(\mathrm{C}^{-6}\right), 56.09\left(\mathrm{OCH}_{3}\right), 56.15\left(\mathrm{OCH}_{3}\right), 59.47\left(\mathrm{C}-4{ }^{\prime}\right), 64.00$ (C-5'), 67.16 (C-3'), 74.96 (C-2'), 104.08, 106.49, 109.63, 122.52, 126.37, 128.30, 128.45, 129.19, $129.27,129.40,129.62,129.74,130.21,135.48,136.57,138.85,148.17,149.42,155.44,180.13$ (C-2"), 206.35 (C-1). Anal. calcd for $\mathrm{C}_{34} \mathrm{H}_{30} \mathrm{~N}_{2} \mathrm{O}_{4}$ : C, 76.96; H, 5.70; N, 5.28. Found: C, 76.82; H, $5.47 ; \mathrm{N}, 5.19 \%$.

5'-Benzyl-5, 6-dimethoxy-2,3-dihydro-4'-(2-methylphenyl)dispiro[1H-indene-2,3'-pyrrolidine-2',3"indoline]-1,2"-dione (4b). IR (KBr) $v_{\max } 3427,1720,1687,1619,1591 \mathrm{~cm}^{-1}$; ${ }^{1} \mathrm{H}-\mathrm{NMR}$ (400 MHz, $\mathrm{CDCl} 3$ ): $\delta_{\mathrm{H}} 1.92\left(\mathrm{~s}, 3 \mathrm{H}, \mathrm{CH}_{3}\right), 2.62\left(\mathrm{~d}, J=18.32 \mathrm{~Hz}, 1 \mathrm{H}, 3-\mathrm{CH}_{2}\right), 2.73\left(\mathrm{~d}, J=18.32 \mathrm{~Hz}, 1 \mathrm{H}, 3-\mathrm{CH}_{2}\right)$, $2.93\left(\mathrm{dd}, J=13.96,8.0 \mathrm{~Hz}, 1 \mathrm{H}, 6{ }^{\prime}-\mathrm{CH}_{2}\right), 3.02\left(\mathrm{dd}, J=13.92,4.4 \mathrm{~Hz}, 1 \mathrm{H}, 6{ }^{\prime}-\mathrm{CH}_{2}\right), 3.70\left(\mathrm{~s}, 3 \mathrm{H}, \mathrm{OCH}_{3}\right)$, $3.80\left(\mathrm{~s}, 3 \mathrm{H}, \mathrm{OCH}_{3}\right), 4.10$ (d, $J=8.8 \mathrm{~Hz}, 1 \mathrm{H}, \mathrm{H}-4$ '), $4.95-5.01$ (m, 1H, H-5'), 6.19 (s, 1H, Ar-H), 6.57 (d, $J=7.32 \mathrm{~Hz}, 1 \mathrm{H}, \mathrm{Ar}-\mathrm{H}), 6.86(\mathrm{t}, J=7.32 \mathrm{~Hz}, 1 \mathrm{H}, \mathrm{Ar}-\mathrm{H}), 6.90(\mathrm{~s}, 1 \mathrm{H}, \mathrm{ArH}), 6.99-7.31(\mathrm{~m}, 9 \mathrm{H}$, Ar-H), 7.36 (d, $J=7.36 \mathrm{~Hz}, 1 \mathrm{H}, \mathrm{Ar}-\mathrm{H}), 8.01(\mathrm{~s}, 1 \mathrm{H}, \mathrm{NH}), 8.04$ (d, $J=8.0 \mathrm{~Hz}, 1 \mathrm{H}, \mathrm{Ar}-\mathrm{H}) .{ }^{13} \mathrm{C}-\mathrm{NMR}$ $\left(100 \mathrm{MHz}, \mathrm{CDCl}_{3}\right): \delta_{\mathrm{C}} 20.33\left(\mathrm{CH}_{3}\right), 35.16(\mathrm{C}-3), 40.45\left(\mathrm{C}-6^{\prime}\right), 55.44(\mathrm{C}-4), 55.95\left(\mathrm{OCH}_{3}\right), 56.04$ $\left(\mathrm{OCH}_{3}\right), 64.85\left(\mathrm{C}-5^{\prime}\right), 66.69\left(\mathrm{C}-3^{\prime}\right), 75.08\left(\mathrm{C}-2^{\prime}\right), 103.96,106.41,109.35,122.51,126.13,126.23$, $126.51,126.69,127.05,127.71,128.23,129.00$, 129.14, 129.66, 130.08, 137.46, 137.91, 138.92, 
140.92, 148.09, 149.27, 155.36, 179.63 (C-2"), 207.10 (C-1). Anal. calcd for $\mathrm{C}_{35} \mathrm{H}_{32} \mathrm{~N}_{2} \mathrm{O}_{4}$ : C, 77.18; $\mathrm{H}$, 5.92; N, 5.14. Found: C, 77.39; H, 5.78; N, 5.02\%.

5'-Benzyl-5,6-dimethoxy-2,3-dihydro-4'-(2-methoxyphenyl)dispiro[1H-indene-2,3'-pyrrolidine-2',3"indoline]-1,2"-dione (4c). IR (KBr) $v_{\max } 3429,1722,1688,1621,1595 \mathrm{~cm}^{-1}$; ${ }^{1} \mathrm{H}-\mathrm{NMR}$ (400 MHz, $\left.\mathrm{CDCl}_{3}\right): \delta_{\mathrm{H}} 2.40-2.54\left(\mathrm{~m}, 2 \mathrm{H}, 3-\mathrm{CH}_{2}\right), 2.91-3.09\left(\mathrm{~m}, 2 \mathrm{H}, 6^{\prime}-\mathrm{CH}_{2}\right), 3.66\left(\mathrm{~s}, 3 \mathrm{H}, \mathrm{OCH}_{3}\right), 3.69(\mathrm{~s}, 3 \mathrm{H}$, $\left.\mathrm{OCH}_{3}\right) 3.87\left(\mathrm{~s}, 3 \mathrm{H}, \mathrm{OCH}_{3}\right), 4.14(\mathrm{~d}, J=9.2 \mathrm{~Hz}, 1 \mathrm{H}, \mathrm{H}-4$ '), 5.02-5.10 (m, 1H, H-5'), 6.10 (s, 1H, Ar-H), $6.53(\mathrm{~d}, J=7.2 \mathrm{~Hz}, 1 \mathrm{H}, \mathrm{Ar}-\mathrm{H}), 6.61(\mathrm{~d}, J=8.0 \mathrm{~Hz}, 1 \mathrm{H}, \mathrm{Ar}-\mathrm{H}), 6.82-7.40(\mathrm{~m}, 11 \mathrm{H}, \mathrm{Ar}-\mathrm{H}), 7.80(\mathrm{~d}$, $J=7.2 \mathrm{~Hz}, 1 \mathrm{H}, \mathrm{Ar}-\mathrm{H}), 8.59$ (s, 1H, NH). ${ }^{13} \mathrm{C}-\mathrm{NMR}\left(100 \mathrm{MHz}, \mathrm{CDCl}_{3}\right): \delta \mathrm{c} 34.72(\mathrm{C}-3), 41.02\left(\mathrm{C}-6^{\prime}\right)$, $53.48\left(\mathrm{C}^{\prime} \mathrm{4}^{\prime}\right), 54.41\left(\mathrm{OCH}_{3}\right), 56.13\left(\mathrm{OCH}_{3}\right), 61.68\left(\mathrm{OCH}_{3}\right), 65.53\left(\mathrm{C}-5^{\prime}\right), 67.15\left(\mathrm{C}-3^{\prime}\right), 75.01\left(\mathrm{C}-2^{\prime}\right)$, $104.11,106.40,109.52,109.79,120.65,122.59,126.16,126.67,127.71,128.15,128.30,128.98$, 129.15, 129.25, 135.42, 139.34, 148.72, 149.09, 155.06, 158.17, 179.63 (C-2"), 208.06 (C-1). Anal. calcd for $\mathrm{C}_{35} \mathrm{H}_{32} \mathrm{~N}_{2} \mathrm{O}$ : : C, 74.98; H, 5.75; N, 5.00. Found: C, 75.21; H, 5.61; N, 5.12\%.

5'-Benzyl-5, 6-dimethoxy-2,3-dihydro-4'-(2-chlorophenyl)dispiro[1H-indene-2,3'-pyrrolidine-2',3"indoline]-1,2"-dione (4d). IR (KBr) $v_{\max } 3422,1716,1684,1620,1595 \mathrm{~cm}^{-1}$; ${ }^{1} \mathrm{H}-\mathrm{NMR}$ (400 MHz, $\left.\mathrm{CDCl}_{3}\right): \delta_{\mathrm{H}} 2.51\left(\mathrm{~d}, J=18.0 \mathrm{~Hz}, 1 \mathrm{H}, 3-\mathrm{CH}_{2}\right), 2.64$ (d, $\left.J=18.0 \mathrm{~Hz}, 1 \mathrm{H}, 3-\mathrm{CH}_{2}\right), 2.96-3.10(\mathrm{~m}, 2 \mathrm{H}$, 6'- $\left.\mathrm{CH}_{2}\right), 3.69\left(\mathrm{~s}, 3 \mathrm{H}, \mathrm{OCH}_{3}\right), 3.81\left(\mathrm{~s}, 3 \mathrm{H}, \mathrm{OCH}_{3}\right), 4.39$ (d, J = 8.8 Hz, 1H, H-4'), 4.92-4.99 (m, 1H, H-5'), 6.30 (s, 1H, Ar-H), 6.60 (d, $J=7.6 \mathrm{~Hz}, 1 \mathrm{H}, \mathrm{Ar}-\mathrm{H}), 6.92-7.47$ (m, 10H, Ar-H), 7.95 (s, 1H, $\mathrm{ArH}), 8.07$ (d, $J=7.6 \mathrm{~Hz}, 1 \mathrm{H}, \mathrm{Ar}-\mathrm{H}), 8.36$ (s, 1H, NH). ${ }^{13} \mathrm{C}-\mathrm{NMR}\left(100 \mathrm{MHz}, \mathrm{CDCl}_{3}\right)$ : $\delta \mathrm{c} 34.77(\mathrm{C}-3)$, $40.78\left(\mathrm{C}-6^{\prime}\right), 56.28\left(\mathrm{OCH}_{3}\right), 56.39\left(\mathrm{OCH}_{3}\right), 61.10\left(\mathrm{C}-4^{\prime}\right), 66.28\left(\mathrm{C}-5^{\prime}\right), 67.16\left(\mathrm{C}-3^{\prime}\right), 75.37\left(\mathrm{C}-2^{\prime}\right)$, $105.27,106.54,107.18,109.66,122.72,126.81,127.95,128.26,128.56,129.08,129.18,129.84$, 131.11, 133.87, 136.13, 137.39, 138.53, 149.39, 149.78, 155.45, 179.60 (C-2"), 207.11 (C-1). Anal. calcd for $\mathrm{C}_{34} \mathrm{H}_{29} \mathrm{ClN}_{2} \mathrm{O}_{4}$ : C, 72.27; H, 5.17; N, 4.96. Found: C, 72.06; H, 5.41; N, 4.87\%.

5'-Benzyl-5, 6-dimethoxy-2,3-dihydro-4'-(3-methoxyphenyl)dispiro[1H-indene-2,3'-pyrrolidine-2',3"indoline]-1,2"-dione (4e). IR (KBr) $v_{\max } 3438,1716,1692,1618,1587 \mathrm{~cm}^{-1}$; ${ }^{1} \mathrm{H}-\mathrm{NMR}$ (400 MHz, $\left.\mathrm{CDCl}_{3}\right): \delta_{\mathrm{H}} 2.69\left(\mathrm{~d}, J=17.6 \mathrm{~Hz}, 1 \mathrm{H}, 3-\mathrm{CH}_{2}\right), 2.88-3.10\left(\mathrm{~m}, 3 \mathrm{H}, 3-\mathrm{CH}_{2}\right.$ and $\left.6{ }^{\prime}-\mathrm{CH}_{2}\right), 3.67(\mathrm{~s}, 3 \mathrm{H}$, $\left.\mathrm{OCH}_{3}\right), 3.72\left(\mathrm{~s}, 3 \mathrm{H}, \mathrm{OCH}_{3}\right) 3.77\left(\mathrm{~s}, 3 \mathrm{H}, \mathrm{OCH}_{3}\right), 4.08(\mathrm{~d}, J=8.8 \mathrm{~Hz}, 1 \mathrm{H}, \mathrm{H}-4), 4.75-4.82(\mathrm{~m}, 1 \mathrm{H}$, H-5'), 6.22 (s, 1H, Ar-H), 6.60 (d, J=8.0 Hz, 1H, Ar-H), 6.71-7.28 (m, 13H, Ar-H), 8.72 (s, 1H, NH). ${ }^{13} \mathrm{C}-\mathrm{NMR}\left(100 \mathrm{MHz}, \mathrm{CDCl}_{3}\right)$ : $\delta \mathrm{C} 34.42(\mathrm{C}-3), 40.28(\mathrm{C}-6), 55.22\left(\mathrm{OCH}_{3}\right), 56.02\left(\mathrm{OCH}_{3}\right), 56.14$ $\left(\mathrm{OCH}_{3}\right), 60.00$ (C-4'), 64.26 (C-5'), 67.14 (C-3'), 74.94 (C-2'), 104.10, 106.65, 109.71, 112.54, 115.79, $122.53,122.74,123.17,126.31,128.10,128.40,129.21,129.36,129.40,138.79,140.45,148.29$, 149.32, 155.52, 159.68, 180.27 (C-2"), 206.44 (C-1). Anal. calcd for $\mathrm{C}_{35} \mathrm{H}_{32} \mathrm{~N}_{2} \mathrm{O}_{5}$ : C, 74.98; H, 5.75; N, 5.00. Found: C, 75.10; H, 5.86; N, 5.08\%.

5'-Benzyl-5, 6-dimethoxy-2,3-dihydro-4'-(3-nitrophenyl)dispiro[1H-indene-2,3'-pyrrolidine-2',3"indoline]-1,2"-dione (4f). IR (KBr) $v_{\max } 3425,1721,1684,1623,1590 \mathrm{~cm}^{-1}$; ${ }^{1} \mathrm{H}-\mathrm{NMR}$ (400 MHz, $\left.\mathrm{CDCl}_{3}\right): \delta_{\mathrm{H}} 2.55\left(\mathrm{~d}, J=17.6 \mathrm{~Hz}, 1 \mathrm{H}, 3-\mathrm{CH}_{2}\right), 2.90\left(\mathrm{~d}, J=17.6 \mathrm{~Hz}, 1 \mathrm{H}, 3-\mathrm{CH}_{2}\right), 2.94-2.98\left(\mathrm{~m}, 1 \mathrm{H}, 6{ }^{\prime}-\mathrm{CH}_{2}\right)$, 3.10-3.19 (m, 1H, 6'- $\left.\mathrm{CH}_{2}\right), 3.73\left(\mathrm{~s}, 3 \mathrm{H}, \mathrm{OCH}_{3}\right), 3.81\left(\mathrm{~s}, 3 \mathrm{H}, \mathrm{OCH}_{3}\right), 3.88\left(\mathrm{~d}, J=8.8 \mathrm{~Hz}, 1 \mathrm{H}, \mathrm{H}-4^{\prime}\right)$, 4.78-4.84 (m, 1H, H-5'), 6.39 (s, 1H, Ar-H), 6.68 (d, J=7.2 Hz, 1H, Ar-H), 6.85-7.86 (m, 10H, Ar-H), 7.67 (d, $J=7.2 \mathrm{~Hz}, 1 \mathrm{H}, \mathrm{Ar}-\mathrm{H}), 7.97$ (d, $J=8.0 \mathrm{~Hz}, 1 \mathrm{H}, \mathrm{Ar}-\mathrm{H}), 8.28$ (s, 1H, ArH), 8.47 (s, 1H, $\mathrm{NH}) .{ }^{13} \mathrm{C}-\mathrm{NMR}\left(100 \mathrm{MHz}, \mathrm{CDCl}_{3}\right): \delta \mathrm{C} 34.52(\mathrm{C}-3), 40.76\left(\mathrm{C}^{\prime} 6^{\prime}\right), 56.12\left(\mathrm{OCH}_{3}\right), 56.18\left(\mathrm{OCH}_{3}\right), 60.09$ 
(C-4'), 65.47 (C-5'), 67.16 (C-3'), 75.15 (C-2'), 104.32, 106.69, 109.70, 122.84, 122.98, 126.41, $126.53,126.78,127.82,128.36,129.18,129.27,136.75,137.93,141.00,141.72,147.74,148.29$, 149.66, 155.90, 179.76 (C-2"), 206.80 (C-1). Anal. calcd for $\mathrm{C}_{34} \mathrm{H}_{29} \mathrm{~N}_{3} \mathrm{O}_{6}$ : C, 70.94; H, 5.08; N, 7.30. Found: C, 70.68; H, 5.25; N, 7.41\%.

5'-Benzyl-5, 6-dimethoxy-2,3-dihydro-4'-(4-methylphenyl)dispiro[1H-indene-2,3'-pyrrolidine-2',3"indoline]-1,2"-dione (4g). IR (KBr) $v_{\max } 3420,1723,1691,1620,1589 \mathrm{~cm}^{-1}$; ${ }^{1} \mathrm{H}-\mathrm{NMR}$ (400 MHz, $\left.\mathrm{CDCl}_{3}\right): \delta_{\mathrm{H}} 2.30\left(\mathrm{~s}, 3 \mathrm{H}, \mathrm{CH}_{3}\right), 2.67\left(\mathrm{~d}, J=17.6 \mathrm{~Hz}, 1 \mathrm{H}, 3-\mathrm{CH}_{2}\right), 2.85-2.94\left(\mathrm{~m}, 2 \mathrm{H}, 3-\mathrm{CH}_{2}\right.$ and $\left.6{ }^{\prime}-\mathrm{CH}_{2}\right)$, $3.07\left(\mathrm{dd}, J=14.0,2.8 \mathrm{~Hz}, 1 \mathrm{H}, 6{ }^{\prime}-\mathrm{CH}_{2}\right), 3.66\left(\mathrm{~s}, 3 \mathrm{H}, \mathrm{OCH}_{3}\right), 3.77\left(\mathrm{~s}, 3 \mathrm{H}, \mathrm{OCH}_{3}\right), 3.81(\mathrm{~d}, J=10.4 \mathrm{~Hz}$, 1H, H-4'), 4.75-4.82 (m, 1H, H-5'), 6.14 (s, 1H, Ar-H), 6.56 (d, J=8.0 Hz, 1H, Ar-H), 6.83-7.29 (m, 13H, Ar-H), $8.48(\mathrm{~s}, 1 \mathrm{H}, \mathrm{NH}) .{ }^{13} \mathrm{C}-\mathrm{NMR}\left(100 \mathrm{MHz}, \mathrm{CDCl}_{3}\right): \delta_{\mathrm{c}} 21.15\left(\mathrm{CH}_{3}\right), 34.24(\mathrm{C}-3), 40.13$ $\left(\mathrm{C}^{\prime} \mathrm{6}^{\prime}\right), 56.01\left(\mathrm{OCH}_{3}\right), 56.14\left(\mathrm{OCH}_{3}\right), 59.44\left(\mathrm{C}-4{ }^{\prime}\right), 63.92\left(\mathrm{C}-5^{\prime}\right), 67.21(\mathrm{C}-3 \mathrm{\prime}), 74.84\left(\mathrm{C}-2^{\prime}\right), 104.07$, $106.61,109.65,122.54,126.31,128.22,128.43,129.14,129.26,129.37,129.60,129.75,130.15$, 135.45, 136.62, 138.87, 148.16, 149.30, 155.46, 180.26 (C-2"), 206.25 (C-1). Anal. calcd for $\mathrm{C}_{35} \mathrm{H}_{32} \mathrm{~N}_{2} \mathrm{O}_{4}$ : C, 77.18; H, 5.92; N, 5.14. Found: C, 77.43; H, 5.74; N, 5.01\%.

5'-Benzyl-5, 6-dimethoxy-2,3-dihydro-4'-(4-methoxyphenyl)dispiro[1H-indene-2,3'-pyrrolidine-2', 3"indoline]-1,2"-dione (4h). IR (KBr) $v_{\max } 3426,1722,1691,1622,1596 \mathrm{~cm}^{-1}$; ${ }^{1} \mathrm{H}-\mathrm{NMR}$ (400 MHz, $\left.\mathrm{CDCl}_{3}\right): \delta_{\mathrm{H}} 2.69\left(\mathrm{~d}, J=17.6 \mathrm{~Hz}, 1 \mathrm{H}, 3-\mathrm{CH}_{2}\right), 2.85-2.96\left(\mathrm{~m}, 2 \mathrm{H}, 3-\mathrm{CH}_{2}\right.$ and 6'-CH 2$), 3.03-3.08(\mathrm{~m}, 1 \mathrm{H}$, 6'- $\left.\mathrm{CH}_{2}\right), 3.71\left(\mathrm{~s}, 3 \mathrm{H}, \mathrm{OCH}_{3}\right), 3.76\left(\mathrm{~s}, 3 \mathrm{H}, \mathrm{OCH}_{3}\right), 3.78\left(\mathrm{~s}, 3 \mathrm{H}, \mathrm{OCH}_{3}\right), 3.85-3.98(\mathrm{~m}, 1 \mathrm{H}, \mathrm{H}-4$ '), 3.67-3.75 (m, 1H, H-5'), 6.29 (s, 1H, Ar-H), 6.57 (d, $J=7.2 \mathrm{~Hz}, 1 \mathrm{H}, \mathrm{Ar}-\mathrm{H}), 6.79-7.62(\mathrm{~m}, 13 \mathrm{H}$, $\mathrm{Ar}-\mathrm{H}), 8.09(\mathrm{~s}, 1 \mathrm{H}, \mathrm{NH}) .{ }^{13} \mathrm{C}-\mathrm{NMR}\left(100 \mathrm{MHz}, \mathrm{CDCl}_{3}\right): \delta \mathrm{c} 34.15(\mathrm{C}-3), 40.22(\mathrm{C}-6 \mathrm{\prime}), 55.27\left(\mathrm{OCH}_{3}\right)$, $56.04\left(\mathrm{OCH}_{3}\right), 56.15\left(\mathrm{OCH}_{3}\right), 59.16\left(\mathrm{C}-4^{\prime}\right), 64.12\left(\mathrm{C}-5^{\prime}\right), 67.26\left(\mathrm{C}-3^{\prime}\right), 74.73\left(\mathrm{C}-2^{\prime}\right), 104.13,106.70$, $109.51,113.88,122.58,126.31,128.30,128.42,129.15,129.35,130.58,131.31,132.42,138.89$, 148.18, 149.31, 155.49, 158.55, 180.01 (C-2"), 206.30 (C-1). Anal. calcd for $\mathrm{C}_{35} \mathrm{H}_{32} \mathrm{~N}_{2} \mathrm{O}$ 5: C, 74.98; H, $5.75 ; \mathrm{N}, 5.00$. Found: C, 74.70; H, 5.96; N, 5.13\%.

5'-Benzyl-5, 6-dimethoxy-2,3-dihydro-4'-(4-chlorophenyl)dispiro[1H-indene-2,3'-pyrrolidine-2',3"indoline]-1,2"-dione (4i). IR (KBr) $v_{\max } 3389,1718,1685,1615,1594 \mathrm{~cm}^{-1}$; ${ }^{1} \mathrm{H}-\mathrm{NMR}(400 \mathrm{MHz}$, $\left.\mathrm{CDCl}_{3}\right): \delta \mathrm{H} 2.68\left(\mathrm{~d}, J=17.6 \mathrm{~Hz}, 1 \mathrm{H}, 3-\mathrm{CH}_{2}\right), 2.89-2.99\left(\mathrm{~m}, 2 \mathrm{H}, 3-\mathrm{CH}_{2}\right.$ and 6'-CH 2$), 3.06-3.10(\mathrm{~m}, 1 \mathrm{H}$, 6'- $\left.\mathrm{CH}_{2}\right), 3.68\left(\mathrm{~s}, 3 \mathrm{H}, \mathrm{OCH}_{3}\right), 3.81\left(\mathrm{~s}, 3 \mathrm{H}, \mathrm{OCH}_{3}\right), 3.95\left(\mathrm{~d}, J=8.8 \mathrm{~Hz}, 1 \mathrm{H}, \mathrm{H}-4{ }^{\prime}\right), 4.72-4.84(\mathrm{~m}, 1 \mathrm{H}$, H-5'), 6.21 (s, 1H, Ar-H), 6.57 (d, J=7.6 Hz, 1H, Ar-H), 6.83-7.45 (m, 13H, Ar-H), 8.40 (s, 1H, NH). ${ }^{13} \mathrm{C}-\mathrm{NMR}\left(100 \mathrm{MHz}, \mathrm{CDCl}_{3}\right): \delta \mathrm{C} 34.30(\mathrm{C}-3), 40.17(\mathrm{C}-6), 56.10\left(\mathrm{OCH}_{3}\right), 56.22\left(\mathrm{OCH}_{3}\right), 59.45\left(\mathrm{C}-4^{\prime}\right)$, 64.04 (C-5'), 67.13 (C-3'), 75.05 (C-2'), 104.12, 106.45, 109.62, 122.47, 126.46, 128.35, 128.43, $129.24,129.31,129.47,129.61,129.80,130.29,135.52,136.61,138.87,148.20,149.46,155.49$, 180.21 (C-2"), 206.63 (C-1). Anal. calcd for $\mathrm{C}_{34} \mathrm{H}_{29} \mathrm{ClN}_{2} \mathrm{O}_{4}$ : C, 72.27; H, 5.17; N, 4.96. Found: C, $72.50 ; \mathrm{H}, 5.02 ; \mathrm{N}, 4.87 \%$.

5'-Benzyl-5, 6-dimethoxy-2,3-dihydro-4'-(2,4-dichlorophenyl)dispiro[1H-indene-2,3'-pyrrolidine-2', 3"'indoline]-1,2"-dione (4j). IR (KBr) $v_{\max } 3394,1721,1682,1614,1583 \mathrm{~cm}^{-1}$; ${ }^{1} \mathrm{H}-\mathrm{NMR}$ (400 MHz, $\left.\mathrm{CDCl}_{3}\right): \delta_{\mathrm{H}} 2.47\left(\mathrm{~d}, J=18.0 \mathrm{~Hz}, 1 \mathrm{H}, 3-\mathrm{CH}_{2}\right), 2.62\left(\mathrm{~d}, J=18.0 \mathrm{~Hz}, 1 \mathrm{H}, 3-\mathrm{CH}_{2}\right), 2.93(\mathrm{dd}, J=14.0$, $\left.5.8 \mathrm{~Hz}, 1 \mathrm{H}, 6{ }^{\prime}-\mathrm{CH}_{2}\right), 2.99$ (dd, $\left.J=14.0,5.8 \mathrm{~Hz}, 1 \mathrm{H}, 6{ }^{\prime}-\mathrm{CH}_{2}\right), 3.72\left(\mathrm{~s}, 3 \mathrm{H}, \mathrm{OCH}_{3}\right), 3.81\left(\mathrm{~s}, 3 \mathrm{H}, \mathrm{OCH}_{3}\right)$, $4.31\left(\mathrm{~d}, J=8.8 \mathrm{~Hz}, 1 \mathrm{H}, \mathrm{H}-4^{\prime}\right), 4.84-4.90$ (m, 1H, H-5'), 6.30 (s, 1H, Ar-H), 6.59 (d, J= 7.2, 1H, Ar-H), 
6.84-7.35 (m, 9H, Ar-H), 7.47 (s, 1H, ArH), $7.60(\mathrm{~d}, J=8.8 \mathrm{~Hz}, 1 \mathrm{H}, \operatorname{Ar}-\mathrm{H}), 8.00(\mathrm{~d}, J=8.8 \mathrm{~Hz}, 1 \mathrm{H}$, $\mathrm{Ar}-\mathrm{H}), 8.40(\mathrm{~s}, 1 \mathrm{H}, \mathrm{NH}) .{ }^{13} \mathrm{C}-\mathrm{NMR}\left(100 \mathrm{MHz}, \mathrm{CDCl}_{3}\right)$ : $\delta \mathrm{c} 34.66(\mathrm{C}-3), 40.88\left(\mathrm{C}-6\right.$ '), $55.67\left(\mathrm{OCH}_{3}\right)$, $56.08\left(\mathrm{OCH}_{3}\right), 64.73\left(\mathrm{C}-4^{\prime}\right), 66.06\left(\mathrm{C}-5^{\prime}\right), 67.15\left(\mathrm{C}^{\prime}-3^{\prime}\right), 75.37\left(\mathrm{C}-2^{\prime}\right), 104.27,106.55,109.70,122.81$, $126.32,126.51,127.17,127.83,128.30,128.73,129.11,129.53,132.19,132.79,136.16,136.60$, 138.27, 141.23, 144.96, 148.02, 149.51, 155.59, 179.31 (C-2"), 206.98 (C-1). Anal. calcd for $\mathrm{C}_{34} \mathrm{H}_{28} \mathrm{Cl}_{2} \mathrm{~N}_{2} \mathrm{O}_{4}$ : C, 68.12; H, 4.71; N, 4.67. Found: C, 68.30; H, 4.94; N, 4.56\%.

\subsection{General Procedure for the Synthesis of $\mathbf{6}$}

To a stirred solution of $\mathbf{4 b}(1 \mathrm{mmol})$ in $5 \mathrm{~mL}$ of dry dichloromethane was added paraformaldehyde ( $1 \mathrm{mmol}$ ), followed by catalytic amount of trifluoroacetic acid at $0{ }^{\circ} \mathrm{C}$. The reaction mixture was allowed to stir overnight and after completion of the reaction, the mixture was washed with water and dried over $\mathrm{Na}_{2} \mathrm{SO}_{4}$. The solvent was removed under reduced pressure to afford $\mathbf{6 b}$ as mixture of products with same $R_{f}$ value, which could not be separated through column chromatography.

\subsection{Cholinesterase Inhibition Assays}

Cholinesterase inhibitory activity of the synthesized compounds was evaluated using the Ellman's microplate assay. For acetylcholinesterase (AChE) inhibitory assay, $140 \mu \mathrm{L}$ of $0.1 \mathrm{M}$ sodium phosphate buffer ( $\mathrm{pH}$ 8) was first added to a 96-wells microplate followed by $20 \mu \mathrm{L}$ of test samples and $20 \mu \mathrm{L}$ of 0.09 units $/ \mathrm{mL}$ acetylcholinesterase enzyme from Electrophoruselectricus (Sigma; St. Louis, MO, USA). After $15 \mathrm{~min}$ of incubation at $25^{\circ} \mathrm{C}, 10 \mu \mathrm{L}$ of $10 \mathrm{mM} \mathrm{5,5'-Dithiobis-2-nitrobenzoic} \mathrm{acid}$ (DTNB) was added into each well, followed by $10 \mu \mathrm{L}$ of acetylthiocholine iodide (14 mM). At $30 \mathrm{~min}$ after the initiation of enzymatic reaction, absorbance of the colored end product was measured using BioTek Power Wave X 340 Microplate Spectrophotometer (BioTek; Winooski, VT, USA) at $412 \mathrm{~nm}$. For butyrylcholinesterase (BChE) inhibitory assay, the same procedure described above was followed, except for the use of enzyme and substrate, instead of which, butyrylcholine esterase from equine serum and $S$-butyrylthiocholine chloride were used. Galanthamine was used as positive control. Test samples and galanthamine were prepared in DMSO at an initial concentration of $1 \mathrm{mg} / \mathrm{mL}$ (1000 ppm). The concentration of DMSO in final reaction mixture was $1 \%$. At this concentration, DMSO has no inhibitory effect on acetylcholinesterase enzyme.

The initial screening was carried out at $10 \mu \mathrm{g} / \mathrm{mL}$ of test samples in $1 \%$ DMSO and each test was conducted in triplicates. Absorbance of the test samples was corrected by subtracting the absorbance of their respective blank. Percentage enzyme inhibition is calculated using the following formula:

$$
\text { Percentage of inhibition }=\frac{\text { (Absorbance of sample - Absorbance of control) }}{\text { Absorbance of control X } 100}
$$

Subsequently, the determination of $\mathrm{IC}_{50}$ was carried out using a set of five concentrations.

\subsection{Molecular Modeling}

Using Glide ${ }^{\mathrm{TM}}$, (version 5.7, Schrödinger, LLC, New York, NY, USA, 2011), most active compound was docked onto the active site of $T c \mathrm{AChE}$ derived from three-dimensional structure of the enzyme complex with anti-Alzheimer's drug, galanthamine (PDB ID: 4EVE). 
Water molecules and hetero groups were deleted from enzyme beyond the radius of $5 \AA$ of reference ligand (galanthamine), resulting protein structure refined and minimized by Protein Preparation Wizard $^{\mathrm{TM}}$ (Version 2014-1, Schrödinger, LLC, New York, NY, USA) using OPLS-2005 force field. Receptor Grid Generation program were used to prepare TcAChE grid and the ligand was optimized by LigPrep $^{\text {TM }}$ (Version 2014-1, Schrödinger LLC, New York, NY, USA) program by using OPLS-2005 force field to generate lowest energy state. Docking stimulations were carried out on bioactive compound, handed in 5 poses per ligand, in which the best pose with highest score was displayed for each ligand.

\section{Conclusions}

In conclusion, the synthesis of a series of novel spiropyrrolidines was accomplished via [bmim] $\mathrm{Br}$ mediated one-pot three component 1,3-dipolar cycloaddition strategy employing a new class of azomethine ylide. Among the spiropyrrolidines $\mathbf{4 a}-\mathbf{j}$, compound $\mathbf{4 f}$ displayed the highest $\mathrm{AChE}$ enzyme inhibitory activity, Molecular-docking analysis, disclosed binding interactions of this compound to the active site residues of the AChE enzyme.

\section{Supplementary Materials}

Copy of FT-IR, ${ }^{1} \mathrm{H}$ and ${ }^{13} \mathrm{C}$ spectra of a representative compound is available on supplementary file. Supplementary materials can be accessed at: http://www.mdpi.com/1420-3049/20/02/2296/s1.

\section{Acknowledgments}

The authors acknowledge the Deanship of Scientific Research at King Saud University for the Research Grant RGP-VPP-026.

\section{Author Contributions}

The contributions of the respective authors are as follows: AIM, RSK, NA-design and synthesis of compounds; AB, YK, VM, MAA, MF-Cholinesterase inhibitory activity and Molecular modeling study. The manuscript was written by RSK and AB.

\section{Conflicts of Interest}

The authors declare no conflict of interest.

\section{References}

1. Terry, A.V.; Buccafusco, J.J. The cholinergic hypothesis of age and Alzheimer's disease-related cognitive deficits: Recent challenges and their implications for novel drug development. J. Pharmacol. Exp. Ther. 2003, 306, 821-827.

2. Cummings, J.L. Alzheimer's disease. N. Engl. J. Med. 2004, 351, 56-67. 
3. Mesulam, M.M.; Guillozet, A.; Shaw, P.; Levey, A.; Duysen, E.G.; Lockridge, O. Acetylcholinesterase knockouts establish central cholinergic pathways and can use butyrylcholinesterase to hydrolyze acetylcholine. Neuroscience 2002, 110, 627-639.

4. Jiang, H.; Zhao, J.; Han, X.; Zhu, S. Stereoselective preparation of 1,2,4-oxadiazole derivatives substituted by pentafluorophenyl by 1,3-dipolar cycloaddition. Tetrahedron 2006, 62, 11008-11011.

5. Coutouli-Argyropoulou, E.; Lianis, P.; Mitakou, M.; Giannoulis, A.; Nowak, J. 1,3-Dipolar cycloaddition approach to isoxazole, isoxazoline and isoxazolidine analogues of $C$-nucleosides related to pseudouridine. Tetrahedron 2006, 62, 1494-1501;

6. Gomes, P.J. S.; Nunes, C.M.; Pais, A.A.C.C.; Pinho e Melo, T.M.V.D.; Arnaut, L.G. 1,3-Dipolar cycloaddition of azomethine ylides generated from aziridines in supercritical carbon dioxide. Tetrahedron Lett. 2006, 47, 5475-5479.

7. Abou-Gharbia, M.A.; Doukas, P.H. Synthesis of Tricyclic Arylspiro Compounds as Potential Antileukemic and Anticonvulsant Agents. Heterocycles 1979, 12, 637-640.

8. Kornett, M.J.; Thio, A.P. Oxindole-3-spiropyrrolidines and -piperidines. Synthesis and local anesthetic activity. J. Med. Chem. 1976, 19, 892-898.

9. Lundahl, K.; Schut, J.; Schlatmann, J.L.M.A.; Paerels, G.B.; Peters, A. Synthesis and antiviral activities of adamantane spiro compounds. 1. Adamantane and analogous spiro-3'-pyrrolidines. J. Med. Chem. 1972, 15, 129-132.

10. Welton, T. Room-Temperature Ionic Liquids. Solvents for Synthesis and Catalysis. Chem. Rev. 1999, 99, 2071-2084.

11. Petkovic, M.; Seddon, K.R.; Rebelo, L.P.N.; Pereira, C.S. Ionic liquids: a pathway to environmental acceptability. Chem. Soc. Rev. 2011, 40, 1383-1403.

12. Yao, Q. $\mathrm{OsO}_{4}$ in ionic liquid [bmim] $\mathrm{PF}_{6}$ : A recyclable and reusable catalyst system for olefin dihydroxylation. remarkable effect of DMAP. Org. Lett. 2002, 4, 2197-2199.

13. Kumar, A.; Pawar, S.S. Converting exo-selective Diels-Alder reaction to endo-selective in chloroloaluminate ionic liquids. J. Org. Chem. 2004, 69, 1419-1420.

14. Fukumoto, K.; Yoshizawa, M.; Ohno, H. Room temperature ionic liquids from 20 natural amino acids. J. Am. Chem. Soc. 2005, 127, 2398-2399.

15. Forsyth, S.A.; Frohlich, U.; Goodrich, P.; Gunaratne, H.Q. N.; Hardacre, C.; McKeown, A.; Seddon, K.R. Functionalised ionic liquids: Synthesis of ionic liquids with tethered basic groups and their use in Heck and Knoevenagel reactions. New J. Chem. 2010, 34, 723-731.

16. Gao, J.; Song, Q.-W.; He, L.-N.; Liu, C.; Yang, Z.-Z.; Han, X.; Li, X.-D.; Song, Q.-C. Preparation of polystyrene-supported Lewis acidic Fe(III) ionic liquid and its application in catalytic conversion of carbon. Tetrahedron 2012, 68, 3835-3842.

17. Narayana Kumar, G.G.K.S.; Aridoss, G.; Laali, K.K. Condensation of propargylic alcohols with indoles and carbazole in $[\mathrm{bmim}]\left[\mathrm{PF}_{6}\right] / \mathrm{Bi}\left(\mathrm{NO}_{3}\right)_{3} \cdot 5 \mathrm{H}_{2} \mathrm{O}$ : A simple high yielding propargylation method with recycling and reuse of the ionic liquid. Tetrahedron Lett. 2012, 53, 3066-3069.

18. Ghahremanzadeh, R.; Ahadi, S.; Bazgir, A. A one-pot, four-component synthesis of $\alpha$-carboline derivatives. Tetrahedron Lett. 2009, 50, 7379-7381.

19. Kabalka, G.W.; Venkataiah, B.; Das, B.C. Allylboration of carbonyl compounds in ionic liquids. Green Chem. 2002, 4, 472-473. 
20. Pingali, S.R.K.; Madhav, M.; Jursic, B.S. An efficient regioselective NBS aromatic bromination in the presence of an ionic liquid. Tetrahedron Lett. 2010, 51, 1383-1385.

21. Wang, X.-S.; Zhou, J.; Yang, K.; Zhang, M.-M. Divergent products obtained from the reactions of salicylaldehyde and 4-hydroxycoumarin in TEBAC- $\mathrm{H}_{2} \mathrm{O}, \mathrm{KF}-\mathrm{Al}_{2} \mathrm{O}_{3}-\mathrm{EtOH}$, and ionic liquid. Synth. Commun. 2010, 40, 3332-3345.

22. Ramachary, D.B.; Narayana, V.V.; Ramakumar, K. Direct ionic liquid promoted organocatalyzed diazo-transfer reactions: diversity-oriented synthesis of diazo-compounds. Tetrahedron Lett. 2008, 49, 2704-2709.

23. Suresh Kumar, R.; Almansour, A.I.; Arumugam, N.; Basiri, A.; Kia, Y.; Kumar, R.R. Ionic Liquid-Promoted Synthesis and Cholinesterase Inhibitory Activity of Highly Functionalized Spiropyrrolidines. Aust. J. Chem. 2014, doi:10.1071/CH14370.

24. Kumar, R.S.; Almansour, A.I.; Arumugam, N.; Osman, H.; Ali, M.A.; Basiri, A.; Kia, Y. An expedient synthesis and screening for antiacetylcholinesterase activity of piperidine embedded novel pentacyclic cage compounds. Med. Chem. 2014, 10, 228-236.

25. Kia, Y.; Osman, H.; Suresh Kumar, R.; Murugaiyah, V.; Basiri, A.; Khaw, K.Y.; Rosli, M.M. An Efficient Ionic Liquid Mediated Synthesis, Cholinesterase Inhibitory Activity and Molecular Modeling Study of Novel Piperidone Embedded $\alpha, \beta$-Unsaturated Ketones. Med Chem. 2014, 10, $512-520$.

26. Kia, Y.; Osman, H.; Suresh Kumar, R.; Basiri, A.; Murugaiyah, V. Synthesis and discovery of highly functionalized mono- and bis-spiro-pyrrolidines as potent cholinesterase enzyme inhibitors. Bioorg. Med. Chem. Lett. 2014, 24, 1815-1819.

27. Kia, Y.; Osman, H.; Suresh Kumar, R.; Basiri, A.; Murugaiyah, V. Ionic liquid mediated synthesis of mono- and bis-spirooxindole-hexahydropyrrolidines as cholinesterase inhibitors and their molecular docking studies. Bioorg. Med. Chem. 2014, 22, 1318-1328.

28. Basiri, A.; Murugaiyah, V.; Osman, H.; Suresh Kumar, R.; Kia, Y.; Hooda, A.; Parsons, R.B. Cholinesterase inhibitory activity versus aromatic core multiplicity: A facile green synthesis and molecular docking study of novel piperidone embedded thiazolopyrimidines. Bioorg. Med. Chem. 2014, 22, 906-916.

29. Kia, Y.; Osman, H.; Kumar, R.S.; Murugaiyah, V.; Basiri, A.; Perumal, S.; Wahab, H.A.; Bing, C.S. Synthesis and discovery of novel piperidone-grafted mono- and bis-spirooxindolehexahydropyrrolizines as potent cholinesterase inhibitors. Bioorg. Med. Chem. 2013, 21, 1696-1707.

30. Basiri, A.; Murugaiyah, V.; Osman, H.; Kumar, R.S.; Kia, Y.; Ali, M.A. Microwave assisted synthesis, cholinesterase enzymes inhibitory activities and molecular docking studies of new pyridopyrimidine derivatives. Bioorg. Med. Chem. 2013, 21, 3022-3031.

31. Kia, Y.; Osman, H.; Kumar, R.S.; Murugaiyah, V.; Basiri, A.; Perumal, S.; Razak, I.A. A facile chemo-, regio- and stereoselective synthesis and cholinesterase inhibitory activity of spirooxindole-pyrrolizine-piperidine hybrids. Bioorg. Med. Chem. Lett. 2013, 23, 2979-2983.

32. Basiri, A.; Murugaiyah, V.; Osman, H.; Kumar, R.S.; Kia, Y.; Awang, K.B.; Ali, M.A. An expedient, ionic liquid mediated multi-component synthesis of novel piperidone grafted cholinesterase enzymes inhibitors and their molecular modeling study. Eur. J. Med. Chem. 2013, 67, 221-229. 
33. Ali, M.A.; Ismail, R.; Choon, T.S.; Kumar, R.S.; Asad, M.; Almansour, A.I.; Yoon, Y.K.; Wei, A.C.; Elumalai, K.; Pandian, S. Alzheimer Diseases: Substituted Spiro [2.3'] Oxindolespiro [3.2"]-5,6Dimethoxy-Indane-1"-One-Indolizine Analogue as Inhibitors of Acetylcholinesterase. Med. Chem. 2012, 2, 7-10.

34. Ali, M.A.; Yar, M.S.; Hasan, M.Z.; Ahsan, M.J.; Pandian, S. Design, synthesis and evaluation of novel 5,6-dimethoxy-1-oxo-2,3-dihydro-1H-2-indenyl-3,4-substituted phenyl methanone analogues. Bioorg. Med. Chem. Lett. 2009, 19, 5075-5077.

35. Castro, A.; Martinez, A. Targeting beta-amyloid pathogenesis through acetylcholinesterase inhibitors. Curr. Pharm. Des. 2006, 12, 4377-4387.

Sample Availability: Samples of the compounds are available from the authors.

(C) 2015 by the authors; licensee MDPI, Basel, Switzerland. This article is an open access article distributed under the terms and conditions of the Creative Commons Attribution license (http://creativecommons.org/licenses/by/4.0/). 